\title{
Building blocks for an adaptive software-based uncertainty estimation
}

\author{
Ivan Poroskun, Daniel Heißelmann, Christian Rothleitner \\ Physikalisch-Technische Bundesanstalt (PTB), Bundesallee 100, Braunschweig, Germany \\ ivan.poroskun@ptb.de
}

\begin{abstract}
Summary:
Measurement uncertainty estimation based on Monte Carlo simulations gains more importance with the ongoing digitalization in metrology. In the field of coordinate metrology, the VCMM is a well-established tool. The concept of this digital twin is transferred to more a general software library, which is consequently applied to develop the Virtual Planck-Balance.
\end{abstract}

Keywords: uncertainty estimation, digital twins, Monte Carlo Simulation, Virtual Planck-Balance, VCMM

\section{Introduction}

In coordinate metrology, the use of the virtual coordinate measuring machine VCMM as a digital twin to calculate the task-specific measurement uncertainty is well established [1]. It is a valuable tool for DAkkS-accredited laboratories as well as in planning and evaluation of measurements in industrial quality control.

PTB's new competence center for virtual measuring instruments (VirtMet) aims at transferring the VCMM concept into other digital twins [2]. The main components and basic principles of the VCMM are identified and generalized for future applications from varying fields of metrology. As a first application, the Virtual Planck-Balance (VPB) will be set up, where the Planck-Balance is a Kibble-Balance for industrial use [3].

Currently, digital twins are independently developed and only very little of actual code is transferred between projects and organizations. Many basic components, such as random number generators (RNG) and routines for uncertainty estimation are likely to be the same and yet they are often implemented anew. From the perspective of software engineering, there is a need for a library which covers most of common needs for the development of digital twins. While there are software libraries available implementing GUM and its supplements (e.g. [6]), the aim of this work focusses on the practical applicability for the development of digital twins.

\section{Proposed structure}

In order to apply the concept of the VCMM to other digital twins it has been analyzed and divided into sub-structures. As a first step, the concept will be transferred to the VPB. For this purpose, a simple software structure (Fig. 1) is proposed. It consists of two main parts: one part which is dependent on a specific project (framed as virtual measurement) and a second part consisting of more general components which can be reused for different digital twins. The latter part consists of measurand(s), stopping rule(s) and a component for uncertainty estimation. A set of distributions for RNGs (visualized as red circles) might also be provided. The whole structure is framed inside a Monte-Carlo simulation loop and only the parametrization of RNGs is performed before the loop starts, whereas the uncertainty is estimated after the simulation.

The base component of the structure is the core: a project specific evaluation algorithm. In case of the VCMM it is represented by a set of fitting algorithms inside the CMM's application software. Preferably, the core already exists at the time the digital twin is created. The models map input parameters into arguments of the core.

The evaluation algorithm provides simulation results in each run of a Monte-Carlo loop. These are saved and observed by stopping rules. After

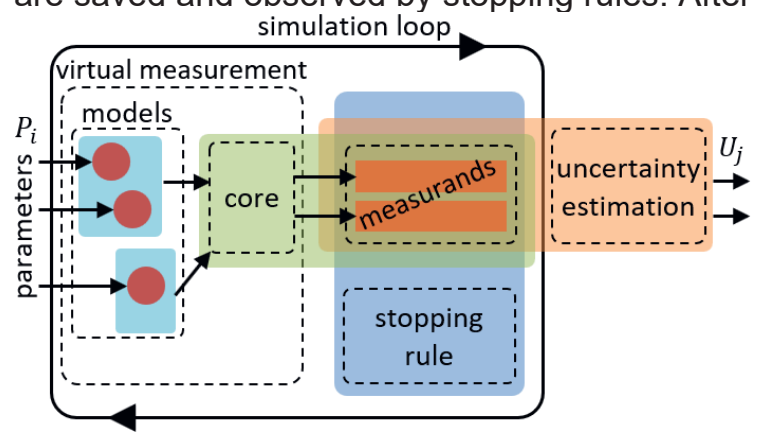

Fig. 1 Proposed structure for digital twins. 
the simulation completes, the values are forwarded into the uncertainty estimation block for further analysis. In the following, we will briefly discuss some aspects of each component.

\section{Stopping rules}

In the simplest case the stopping rule for termination of a Monte-Carlo simulation is given by a fixed number of runs $N$ which is known a priori. If $N$ is chosen too low, the desired numerical tolerance of the result might not be achieved. For this scenario GUM S1 [3] includes an adaptive Monte-Carlo (MC) procedure. As it is done in [4] some improvements of adaptive MC are added over time. The criterion for stopping rule components must be interchangeable with minimal effort to fulfill current preferences of the developer.

\section{Measurands}

Simple measurands are realized as vectors containing simulated values. Besides the individual simulation results, the measurands also contain meta data necessary for the evaluation of the stopping rule and uncertainty estimation components.

\section{Uncertainty estimation}

This component implements algorithms for uncertainty estimation from GUM S1 [3]. The implementation details follow the specifications from [6].

\section{Application to Virtual Planck-Balance}

As all software components common to various digital twins can be covered by the supporting software library, for the devolvement of a new digital twin only the components specific to the individual application must be created.

In a first application, the feasibility of the approach shall be tested by transferring it to the Planck-Balance, thus creating the Virtual Planck-Balance. The core contains the measurement equation of the PB for a single measurement described by [5]

$$
m=\frac{U_{\text {dynamic }} \cdot U_{\text {static }}}{v_{\text {dynamic }} \cdot g \cdot R_{\text {static }}},
$$

where $m$ denotes the mass, $U_{\text {dynamic }}$ and $U_{\text {static }}$ are voltages, $v_{\text {dynamic }}$ is the velocity, $g$ is the local acceleration due to gravity, and $R_{\text {static }}$ is the electrical resistance. The measurement equation (1) can be viewed as a tree-like structure where each of the five arguments is placed onto a separate node of the tree. Each argument is implemented inside a separate class with either a constant value, where no uncertainty of the argument is to be considered, a single RNG or with a complex combination of multiple physical models. The encapsulation of each argument provides the advantage of hiding possible complex details and making the overall model extensible and easy to comprehend.

\section{Conclusion}

A structure is proposed to enable users to create a digital twin of any complex measurement setup. The approach is based on the well-established VCMM, from which a versatile software library containing building blocks for future digital twins is derived. Thus, for any specific application, the building blocks can be used and only the individual models have to be developed. In a next step, the approach will be adapted to the Planck-Balance.

\section{References}

[1] D. Heißelmann, M. Franke, K. Rost, K. Wendt, T. Kistner, and C. Schwehn, Determination of measurement uncertainty by Monte Carlo simulation, Advanced Mathematical and Computational Tools in Metrology and Testing XI, 192-202, (2018); doi: 10.1142/9789813274303_0017.

[2] S. Eichstädt et al., Metrology for virtual measuring systems: new competence centre "VirtMet" at PTB, MathMet2019, Lisbon, Portugal, (2019).

[3] JCGM, Evaluation of measurement data - Supplement 1 to the "Guide to the expression of uncertainty in measurement" - Propagation of distributions using a Monte Carlo method: JCGM 104:2009.

[4] G. Wübbeler, P. M. Harris, M. G. Cox, and C. Elster, A two-stage procedure for determining the number of trials in the application of a Monte Carlo method for uncertainty evaluation, Metrologia, 47(3), 317-324, (2010); doi: 10.1088/00261394/47/3/023.

[5] C. Rothleitner et al., The Planck-Balance - A selfcalibrating precision balance for industrial applications, Engineering for a Changing World, p. 11, (2017).

[6] M. Cox, P. Harris, and I. Smith, Software specifications for uncertainty evaluation, National Physical Laboratory (NPL), NPL Report MS 7, (2010). 